\title{
Coexisting Sources of False-Positive Reflux on a Direct Radionuclide Cystography Scan
}

\author{
Mohsen Qutbi, Ali Asadi, and Isa Neshandar Asli \\ Department of Nuclear Medicine, Taleghani Educational Hospital, School of Medicine, Shahid Beheshti University of Medical \\ Sciences, Tehran, Iran
}

\begin{abstract}
Direct radionuclide cystography is currently a popular method for evaluation of vesicoureteral reflux, despite its pitfalls and drawbacks in producing false-positive results. In this article, we present a case with 2 sources of false-positive reflux on a direct radionuclide cystography scan.
\end{abstract}

Key Words: false positive reflux; direct radionuclide cystography; artifact

J Nucl Med Technol 2019; 47:177-178

DOI: 10.2967/jnmt.118.222075

$\mathbf{T}$ here are a few sources of false-positive results on direct radionuclide cystography scans, of which some are well described in the literature (1). In this article, we present 2 sources of false-positive reflux on direct radionuclide cystography in a single patient. One resulted from systemic absorption of pertechnetate from inflamed bladder mucosa, as was previously described in the literature (2); the other related to an equipment error producing a picture-in-picture artifact.

\section{CASE REPORT}

A 9-y-old girl with a history of reflux was referred for a direct radionuclide cystography scan. After obtaining informed consent, we performed the scan according to standard protocol, with $20 \mathrm{MBq}$ of pertechnetate injected through a Foley catheter placed into the bladder. A singlehead Genesys Epic ADAC $\gamma$-camera was used. During dynamic imaging, a small spot appeared above the bladder on the right side from the beginning to the end. On closer inspection, synchronous changes in intensity were noted between the spot and the bladder. Later in the filling phase, a gradually increasing accumulation of activity in the left renal pelvis was detected, along with transient visualization of the upper ureter thereafter (Fig. 1A). Because we thought

\footnotetext{
Received Oct. 18, 2018; revision accepted Dec. 17, 2018.

For correspondence or reprints contact: Mohsen Qutbi, Department of Nuclear Medicine, Taleghani Hospital, Yaman St., Velenjak, Tehran 1985711151, Iran.

E-mail: mohsen.qutbi@sbmu.ac.ir, mohsen.qutbi@gmail.com

Published online Feb. 15, 2019.

COPYRIGHT (C) 2019 by the Society of Nuclear Medicine and Molecular Imaging.
}

these patterns atypical of vesicoureteral reflux, and to see whether the activity in the left renal pelvis might be excreted by the kidney after systemic absorption of pertechnetate through inflamed bladder mucosa, a thyroid view was obtained. On it, uptake in the salivary glands and thyroid was evident (Fig. 2A). On a repeat scan with ${ }^{99 \mathrm{~m} T c-s u l f u r}$ colloid $2 \mathrm{~d}$ later (Fig. 1B), no activity was evident in the left renal pelvis, but similar to the previous scan, the spot above the bladder was persistent. This time, the thyroid showed no uptake. To exclude the possibility of an undiagnosed renal pelvis with considerable reflux or even a bladder diverticulum, a ${ }^{99} \mathrm{~m} \mathrm{Tc}-$ diethylenetriaminepentaacetic acid renal scan (Fig. 1C) was then conducted, which demonstrated no corresponding abnormality. Because of recent repair of the $\gamma$-camera detector, we thought this result might be an error caused by the camera hardware or even software. Thus, a point source was placed on the scanning table and a spot view was acquired. Interestingly and unexpectedly, a smaller spot with much lower intensity, similar to the index source in shape, appeared above the point on the right (Figs 2B and 2C).

\section{DISCUSSION}

Despite the high sensitivity and favorable dosimetry of a direct radionuclide cystography scan, careful consideration of its pitfalls and possible sources of false-positive reflux is of critical importance (1). One of the pitfalls of direct radionuclide cystography using pertechnetate is systemic absorption from an inflamed mucosa or in an augmented bladder-findings that may pose a significant source of confusion to the interpreter. Thereby, the use of pertechnetate is discouraged even in patients who are not in an active phase of inflammation $(1,2)$. Another problem, which is less known to clinicians, is the mentioned artifact in our patient. The camera used for this scan recently had an error in count detection as a result of a malfunctioning event-processor board. This board had therefore been replaced by another one, which had a minor error as well. This hardware item in $\gamma$-camera detectors processes the events in terms of energy and position. A faulty processor may erroneously localize the events and, as in our patient, cause distortion in the image resulting from a smaller picture appearing in a fixed location in the main picture, or the so-called picture-in-picture artifact. 


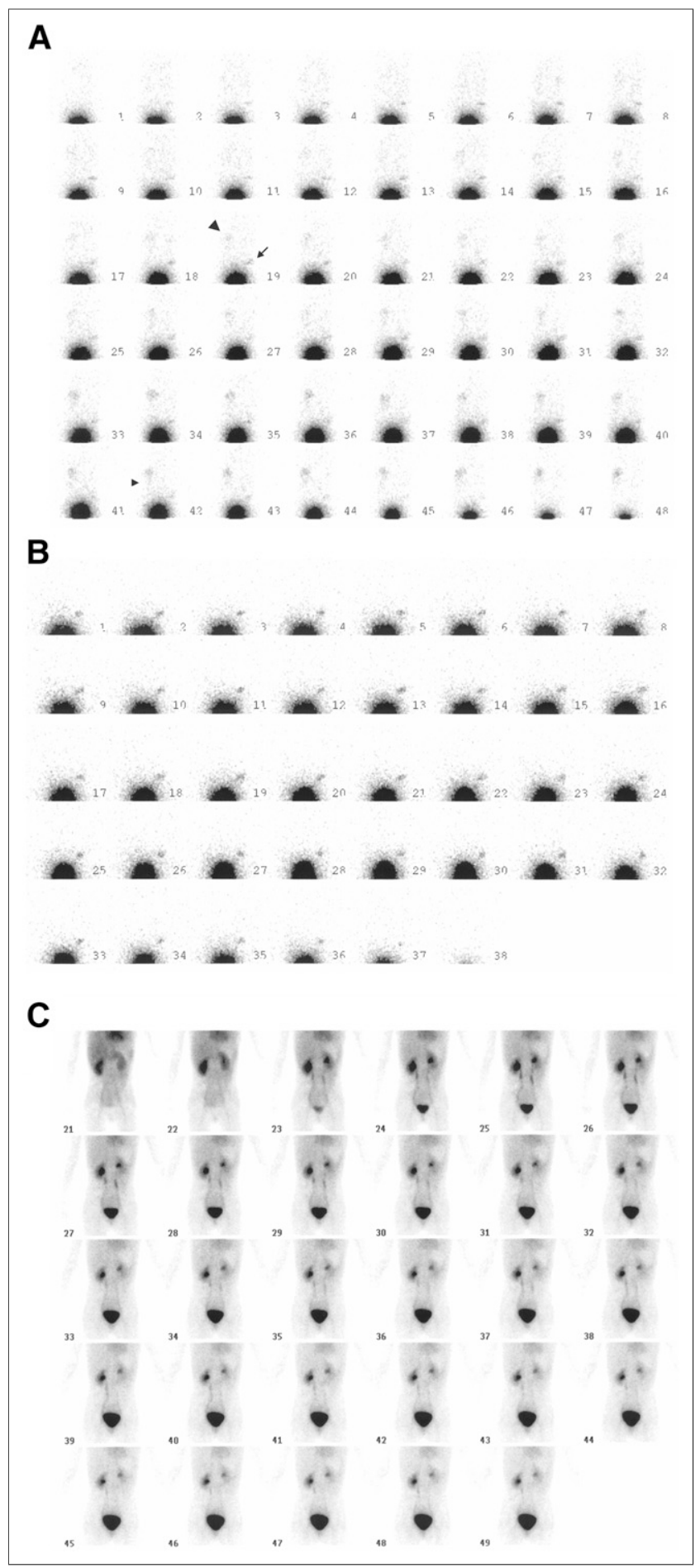

FIGURE 1. (A) Filling and voiding phases of direct radionuclide cystography scan from posterior view. Spot (arrow) appeared from beginning to end of study. On left side, activity is seen in renal pelvis (large arrowhead) and ureter (small arrowhead). (B) Filling and voiding phases of repeat direct radionuclide cystography scan showed no activity in left renal pelvis but persistent focal activity in pelvis above bladder. (C) Dynamic posterior $99 \mathrm{mTc}$-diethylenetriaminepentaacetic renal scan with standard protocol did not show any corresponding abnormality in pelvis.

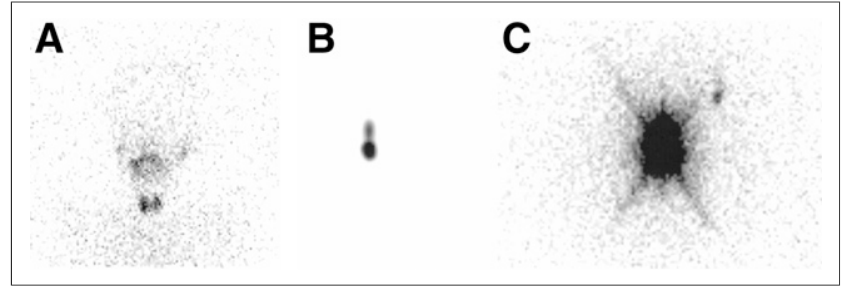

FIGURE 2. (A) Anterior view of head and neck. Uptake in salivary glands and thyroid implies presence of pertechnetate in circulation after absorption through inflamed bladder mucosa. (B and $C$ ) Spot view of point source without $(B)$ and with $(C)$ brightness enhancement. With increase in brightness, another smaller spot became visible above and to right of point source, in location similar to that on patient scan.

\section{CONCLUSION}

Having enough knowledge about the various potential sources of artifacts, either related to patient or equipment, on direct radionuclide cystography scans is necessary for both clinicians and technologists.

\section{DISCLOSURE}

No potential conflict of interest relevant to this article was reported.

\section{REFERENCES}

1. Mandell GA, Eggli DF, Gilday DL, et al. Procedure guideline for radionuclide cystography in children. J Nucl Med. 1997;38:1650-1654.

2. Lowe B, Barnes WF, Leonard JC. Absorption of Tc-99m pertechnetate from the augmented bladder during direct nuclear cystography. Clin Nucl Med. 1989;14: $347-349$. 